\title{
Internações por Doenças Crônicas Não Transmissíveis dos Sistemas Musculoesquelético e Nervoso no envelhecimento
}

\author{
Interventions for Chronic Non-Communicable Diseases of \\ the Musculoskeletal and Nervous Systems in Aging
}

\begin{abstract}
Como citar este artigo:
MORO, MARIANE I. B.; DALENO-

CARE, JÉSSICA F.; MARQUES,

CLANDIO T.; MIRANDA, FERNANDA

A. C. de; Internações por Doenças

Crônicas Não Transmissiveis dos

Sistemas Musculoesquelético

e Nervoso no envelhecimento.

Revista Saúde (Sta. Maria). 2021 ;

$47(1)$
\end{abstract}

\section{Autor correspondente:}

Nome: Mariane Inês Bolson Moro

E-mail: mariane_moro@hotmail.com

Telefone: (55) 999710026

Formação Profissional: Fisiotera-

peuta pela Universidade Federal

de Santa Maria (UFSM), Santa

Maria, RS, Brasil

Filiação Institucional: Hospital Nossa Senhora de Pompéia e Hospital Geral, Caxias do Sul, RS,

Brasil

Endereco para correspondência:

Rua: Feijó Junior n’: 470 - apto

303

Bairro: São Pelegrino

Cidade: Caxias do Sul

Estado: RS

CEP: 95034160

Data de Submissão:

08/07/2020

Data de aceite:

16/04/2021

Conflito de Interesse: Não há conflito de interesse

\section{(c) BY-NC-ND}

\begin{abstract}
Mariane Inês Bolson Moro, Jéssica Franco Dalenogare, Clandio Timm Marques, Fernanda Alves Carvalho de Miranda

\section{RESUMO}

Introdução: As transformações sociais e econômicas pelas quais o Brasil vem passando acabam por alterar também o perfil da incidência de doenças e as causas de internação hospitalar. A alteração também ocorre sobre a expectativa de vida, que aumenta, repercutindo no processo de envelhecimento. Em países que se encontram em transição epidemiológica, como este, pode estar associado à maior prevalência e incidência de Doenças Crônicas Não Transmissiveis (DCNT). Com isso, torna-se relevante analisar o curso dessas doenças com vistas à aplicação de políticas preventivas e planejamento assistencial. Objetivo: Descrever e analisar o comportamento da série histórica das internações por DCNT que acometem o sistema musculoesquelético e o sistema nervoso segundo registro de Autorização de Internação Hospitalar (AlH) em Hospital Escola do sul do país, por grupos etários no processo de envelhecimento, com base na perspectiva de Sahrmann (2014), destacando as DCNT do sistema musculoesquelético e sistema nervoso. Métodos: Trata-se de um estudo observacional, de agregados de séries temporais com dados secundários, publicados pelo Ministério da Saúde de forma online pelo DATASUS. Resultados: Foi possível observar que há maior número de internações por DCNT dos sistemas musculoesquelético e nervoso nas faixas etárias mais jovens. Considerações finais: 0 padrão de maior número das internações nas faixas etárias iniciais, em comparação com as faixas etárias mais velhas, implica na veracidade de que o modelo de sistema do movimento humano apresentado por Sahrmann pode ser um dos caminhos para mais estudos sobre a prevalência de DCNT no envelhecimento.
\end{abstract}

PALAVRAS-CHAVE: Hospitalização; Doenças Crônicas; Envelhecimento.

\section{ABSTRACT}

Introduction: The social and economic transformations that Brazil has been going through also change the profile of the incidence of diseases and the causes of hospitalization. The change also occurs in life expectancy, which increases, affecting the aging process. In countries that are in epidemiological transition, such as this one, it may be associated with a higher prevalence and incidence of Chronic Noncommunicable Diseases (CNCD). Thus, it becomes relevant to analyze the course of these diseases in order to apply preventive policies and care planning. Objective: To describe and analyze the behavior of the historical series of admissions for CNCD that affect the musculoskeletal system and the nervous system according to the Hospitalization Authorization Record (AlH) in a School Hospital in the south of the country, by age groups in the aging process, with based on the perspective of Sahrmann (2014), highlighting the CNCD of the musculoskeletal and nervous systems. Methods: This is an observational study, of time series aggregates with secondary data published by the Ministry of Health online by DATASUS. Results: It was possible to observe that there is a greater number of admissions for CNCD of the musculoskeletal and nervous systems in the younger age groups. Final considerations: The pattern of greater number of hospitalizations in the initial age groups compared to the older age groups, implies the veracity that the human movement system model presented by Sahrmann can be one of the paths for further studies on the prevalence of CNCD in aging. 


\section{INTRODUÇÃO}

Atransição epidemiológica no Brasil implica em grandes desafios no que se refere ao envelhecimento populacional em decorrência do aumento na expectativa de vida da população idosa ${ }^{1,2}$. Cabe ressaltar que existe uma relação direta entre o envelhecimento e o desenvolvimento das Doenças Crônicas Não Transmissíveis (DCNT), visto que elas são doenças de longa duração e que tendem a surgir com o passar dos anos ${ }^{1,3}$.

A imobilidade física está entre os fatores de risco mais relevantes para o aumento da prevalência das DCNT no processo de envelhecimento ${ }^{4}$. Tal fato pode comprometer a qualidade de vida do indivíduo e acarretar graus de incapacidade significativos. Assim, essas doenças tornam-se um desafio na saúde pública, tanto pelas taxas de mortalidade e morbidade quanto pelas questões econômicas do país. Isso porque o acometimento por essas doenças tem relação direta com o aumento das internações hospitalares, com os tratamentos medicamentosos e na reabilitação desses indivíduos, corroborando com o aumento dos gastos na atenção à saúde de alta complexidade do Sistema Único de Saúde (SUS) ${ }^{1}$.

Contudo, as DCNT podem ser prevenidas com aprimoramento de ações de promoção de saúde e prevenção de doenças, e também por meio da investigação do curso das patologias ao longo dos anos de tal forma que fomente 0 fortalecimento dessas ações ${ }^{3}$. Nesse contexto a atuação da fisioterapia torna-se importante, considerada a relevância do trabalho da categoria no que tange à função do sistema de movimento do corpo humano a fim de reduzir as incapacidades que podem vir a afetar pessoas de diversas faixas etárias ${ }^{5}$. Segundo Sahrmann ${ }^{6}$ o sistema do movimento humano pode ser dividido em sistemas efetores, o qual corresponde ao musculoesquelético e nervoso, e sistemas de apoio, no caso cardiovascular, respiratório e endócrino, sendo que eles atuam de forma conjunta, a fim de promover o movimento.

Dessa forma, o presente estudo objetivou descrever e analisar o comportamento da série histórica das internações por DCNT que acometem os sistemas efetores, com base na perspectiva de Sahrmann ${ }^{6}$, segundo registro de Autorização de Internação Hospitalar (AlH) em hospital público, por grupos etários no processo de envelhecimento.

\section{MÉTODO}

Trata-se de um estudo observacional, de agregados de séries temporais com dados secundários publicados pelo Ministério da Saúde de forma online pelo DATASUS (www.datasus.gov.br) conforme a AlH segundo diagnóstico de Patologias e Distúrbios Musculoesqueléticos e Nervosos pertencentes aos capítulos do CID-10 XIII (doenças inflamatórias do sistema nervoso central, atrofias sistêmicas que afetam, principalmente, o sistema nervoso central, doenças extrapiramidais e transtornos dos movimentos, outras doenças degenerativas do sistema nervoso, doenças desmielinizantes do sistema nervoso central, transtornos episódicos e paroxísticos, transtornos dos nervos, das raízes e dos plexos nervosos, polineuropatias e outros transtornos do sistema nervoso periférico, doenças da junção mioneural e 
dos músculos, paralisia cerebral e outras síndromes paralíticas, outros transtornos do sistema nervoso) e VI (artropatias, poliartropatias inflamatórias, artroses, outros transtornos articulares, doenças sistêmicas do tecido conjuntivo, dorsopatias deformantes, dorsopatias, espondilopatias, outras dorsopatias, transtornos musculares, transtornos dos tecidos moles, transtornos das sinóvias e dos tendões, outros transtornos dos tecidos moles, transtornos da densidade e da estrutura óssea, osteopatias e condropatias, outras osteopatias, condropatias, outros transtornos do sistema osteomuscular e do tecido conjuntivo), respectivamente. A amostra foi constituída pela população registrada por internação no Hospital Universitário de Santa Maria (HUSM), na cidade de Santa Maria, no Estado do Rio Grande do Sul, no período de 2008 a 2015. O período foi delimitado entre 2008 a 2015 devido à disponibilidade dos parâmetros selecionados para a pesquisa, visto que em 2008 o DATASUS finalizou a modificação e atualização em seu sistema, proporcionando mais itens disponíveis para a seleção da pesquisa. Ainda, o estudo é delimitado até 2015 visto que a publicação dos dados no sistema até a data mencionada, e, também, visando respeitar os dois anos de revisão das postagens feitas pelo site.

Foram identificadas as variáveis quanto à faixa etária (40 a 49 anos, 50 a 59 anos, 60 a 69 anos, 70 a 79 anos e 80 anos ou mais de idade), com a finalidade de observar o processo de senescência, notificados no DATASUS segundo a $\mathrm{AlH}$, incluindo ambos os sexos (feminino e masculino), assim como todas as raças/cores (branca, preta, parda, amarela e indígena). A escolha da idade de início à análise, 40 anos, foi realizada por compreendermos que nos 20 anos que antecedem a velhice, os hábitos de vida e histórico de patologias (aqui representadas pelas causas de internação hospitalar) que os acompanham são decisivos para o envelhecimento saudável e ativo.

Dessa forma, foram utilizadas informações sobre AlH conforme segundo diagnóstico referente aos capítulos supracitados, provenientes do Sistema de Informações Hospitalares do SUS (SIH-SUS), um banco de dados secundários, disponibilizado pelo DATASUS, disponível para acesso online por toda população. Esse banco contém informações de todas as internações hospitalares realizadas por meio das AlH, documento obrigatório nas internações realizadas pelo SUS.

Ao acessar o sistema DATASUS foram selecionados: Informações de Saúde (TABNET) Epidemiológicas e Morbidade; Morbidade Hospitalar do SUS - por local de internação - Rio Grande do Sul; Município: 431690 Santa Maria; Estabelecimento: 2244306 HUSM HOSPITAL UNIVERSITARIO DE SANTA MARIA; Regime: Público; Capítulo CID-10 (já referidos, um a um); Faixa Etária (já referidas, uma a uma); Sexo: Masc, Fem; Cor/raça: Branca, Preta, Parda, Amarela, Indígena; Período (anos considerados e já citados).

Não houve necessidade de submissão do trabalho ao Comitê de Ética em Pesquisa da Universidade Federal de Santa Maria (UFSM) por se tratar de uma pesquisa que utiliza banco de dados secundários de domínio público disponível online pelo DATASUS e, dessa forma, não havendo variáveis que possibilitem a identificação dos sujeitos da pesquisa.

Para a análise estatística, foi utilizado o teste de Shapiro-Wilk para verificação da normalidade. Na sequência 
foi aplicado o teste Wilcoxon. As diferenças foram consideradas significativas para um $p<0,05$. 0 software IBM SPSS Versão 23 foi utilizado como ferramenta computacional para a análise estatística dos dados.

\section{RESULTADO}

Os resultados da comparação do número de internações por doenças do sistema musculoesquelético e sistema nervoso entre as faixas etárias nos anos de 2008 a 2015 estão expressos na Tabela 1. É possível verificar que no ano de 2008 a faixa etária de 40-49 anos apresenta maior quantidade de internações em comparação com a faixa etária de 70-79 anos. Nos anos de 2009 e 2010 é observado que, na faixa etária de 40-49 anos, há maior número de internações do que a faixa etária de 80 anos ou mais.

Já nos anos de 2011, 2012 e 2013 não houve uma diferença significativa em relação ao número de internações quando analisadas as 5 faixas etárias.

No ano de 2014 foi verificado que, na faixa etária de 80 anos ou mais, temos a menor quantidade de internações por patologias relacionadas aos sistemas musculoesquelético e nervoso, quando comparado com as faixas 50-59 anos e 60-69 anos pelas mesmas causas.

Em 2015 é visto que na faixa etária de 80 anos ou mais é significativamente menor o número de internações em comparação a todas as demais faixas etárias. Além disso, é demonstrado que na faixa de 40-49 anos e 70-79 anos há menor número de internações quando comparada à faixa etária de 50-59 anos.

Tabela 1 - Comparação do número de internações por doenças do sistema musculoesquelético e sistema nervoso entre as faixas etárias nos respectivos anos de 2008, 2009, 2010, 2011, 2012, 2013, 2014 e 2015.

\begin{tabular}{|c|c|c|c|c|c|}
\hline & 40-49 anos & 50-59 anos & $60-69$ anos & $70-79$ anos & 80 anos ou mais \\
\hline 2008 & $0.3462 \pm 0.7452^{c}$ & $0.1538 \pm 0.3679$ & $0.2692 \pm 0.6668$ & $0.1154 \pm 0.5883^{\mathrm{a}}$ & $0.0769 \pm 0.2717$ \\
\hline 2009 & $0.6538 \pm 1.5733^{\mathrm{e}}$ & $0.3462 \pm 0.8918$ & $0.2692 \pm 0.6668$ & $0.3462 \pm 1.1293$ & $0.0385 \pm 0.1961^{a}$ \\
\hline 2010 & $0.6154 \pm 1.1688^{\mathrm{e}}$ & $0.3846 \pm 0.8979$ & $0.3077 \pm 1.0107$ & $0.2692 \pm 1.1852$ & $0.0769 \pm 0.2717^{\mathrm{a}}$ \\
\hline 2011 & $0.4615 \pm 1.1741$ & $0.2692 \pm 0.8274$ & $0.3077 \pm 1.3790$ & $0.1154 \pm 0.4315$ & $0.0385 \pm 0.1961$ \\
\hline 2012 & $0.0385 \pm 0.1961$ & $0.0385 \pm 0.1961$ & $0.1538 \pm 0.4641$ & $0.0385 \pm 0.1961$ & $0 \pm 0$ \\
\hline 2013 & $0.1154 \pm 0.4315$ & $0.0385 \pm 0.1961$ & $0.0385 \pm 0.1961$ & $0 \pm 0$ & $0 \pm 0$ \\
\hline 2014 & $0.1923 \pm 0.4915$ & $0.5 \pm 1.1747^{\mathrm{e}}$ & $0.3077 \pm 0.6177^{\mathrm{e}}$ & $0.1923 \pm 0.4019$ & $0.0385 \pm 0.1961^{b c}$ \\
\hline 2015 & $0.4231 \pm 0.7027^{\text {be }}$ & $1.5385 \pm 3.7654^{\text {ade }}$ & $0.6154 \pm 0.9414^{\mathrm{e}}$ & $0.4615 \pm 1.0288^{\text {be }}$ & $0.0769 \pm 0.2717^{\mathrm{abcd}}$ \\
\hline
\end{tabular}


Os valores são representados como Média \pm Desvio padrão. Letras minúsculas representam diferenças na mesma faixa etária, em diferentes anos. $a=40-49$ anos, $b=50-59$ anos, $c=60-69$ anos, $d=70-79$ anos, $e=80$ anos ou mais. As diferenças foram consideradas significativas para um $p<0,05$.

\section{DISCUSSÃO}

Por meio dos resultados obtidos foi possível verificar que os índices de internação hospitalar por DCNT do sistema musculoesquelético e do sistema nervoso diminuem com o passar da idade. Com isso, pode-se inferir que o modelo do sistema do movimento humano desenvolvido pela autora Sahrmann ${ }^{6}$ pode se apresentar como embasamento para o estudo do envelhecimento e a sua relação com o acometimento por DCNT.

Segundo a autora, o sistema do movimento humano envolve a complexidade da interação entre os sistemas que compõem o organismo. O sistema musculoesquelético, juntamente ao sistema nervoso, é considerado sistema efetor quanto à mobilidade e funcionalidade do corpo. Já os sistemas cardiovascular, respiratório e endócrino apresentam como função dar apoio aos sistemas efetores. Com isso, são vistas uma dependência e influência de um sistema sobre o outro. Assim, a atividade física apresenta-se necessária tanto para a manutenção saudável do sistema motor e nervoso, quanto para o sistema cardiovascular, respiratório e endócrino e todo o funcionamento orgânico, evitando o acometimento de doenças ${ }^{6}$.

Em um estudo realizado em 2007 foi possível observar que as doenças musculoesqueléticas apresentam maior incidência em idosos com mais de 70 anos $^{2}$. Isso implica que, com o avanço da idade, a incapacidade física se agrava. Em contrapartida, no presente estudo foi possível perceber que os índices de internação por doenças musculoesqueléticas e, além delas, as do sistema nervoso, foram maiores em indivíduos entre 40 e 49 anos quando comparados aos indivíduos de 70 a 79 anos no ano de 2008. Cabe ressaltar que essa estimativa prosseguiu nos anos de 2009 e 2010 com idosos com 80 anos ou mais.

Alves et al. ${ }^{7}$ observou que em idosos em uma média de idade entre 71 e 72 anos, a prevalência de DCNT que interferiam nas suas atividades instrumentais de vida diária de forma significativa, foram a hipertensão arterial em $39 \%$, a doença cardíaca em 82\%, a artropatia em 59\% e a doença pulmonar em 50\%. Isso implica na influência que as doenças crônicas apresentam sobre a capacidade funcional dos idosos. Tal fato pode ser correlacionado com o modelo do sistema do movimento humano apresentado por Sahrmann ${ }^{6}$, isso porque segundo o estudo de Alves et al. ${ }^{7}$, as principais doenças crônicas que limitam a capacidade funcional dos indivíduos são doenças que acometem a manutenção e o sustento do movimento humano pertencentes aos sistemas de apoio ao sistema do movimento humano, exceto a artropatia, que faz parte das doenças musculoesqueléticas. Tendo isso em mente, pode-se inferir que, com o avanço da idade, 0 principal acometimento no processo de envelhecimento, as doenças musculoesqueléticas e do sistema nervoso perdem 
espaço em relação às cardiovasculares e pulmonares. Essa evidência torna a hipótese deste estudo positiva, já que as internações por doenças musculoesqueléticas e do sistema nervoso diminuíram com o envelhecimento.

Cabe ressaltar que o movimento humano é uma atividade individual indispensável tanto para a manutenção da vida e saúde quanto para a interação social e mobilidade espacial. O prejuízo na mobilidade física pode manifestarse súbita ou lentamente, tendo extensões e duração específicas em cada caso. Com isso, a imobilidade acaba por acarretar o desenvolvimento ou agravamento de diversas comorbidades físicas e biológicas, assim como repercussões psicológicas e sociais ${ }^{8,9,10}$. Essas condições e repercussões refletem nos índices de internações hospitalares, como mostra o estudo de Chibante et al. ${ }^{11}$ o qual aponta que pacientes com média de idade entre 61 e 70 anos e que estão internados por conta do desenvolvimento de DCNT ficam internados, em média, 19 a 21 dias. Além disso, afirmam que a presença das DCNT somada às comorbidades associadas estão diretamente ligadas ao tempo maior de permanência no hospital e ao aumento das recidivas desses indivíduos com uma nova internação.

Pereira, Nogueira e da Silva ${ }^{12}$ apontam em um estudo que as DCNT são as patologias que mais interferem na qualidade de vida na terceira idade, sendo que as principais são as doenças cardiovasculares numa faixa etária de idosos acima de 80 anos. Esse fato corrobora a hipótese de que o modelo apresentado por Sahrmann ${ }^{6}$ pode descrever o curso das DCNT no envelhecimento, ou seja, as doenças pertencentes aos sistemas efetores do movimento levam tardiamente ao desenvolvimento de doenças que acometem os sistemas de apoio ao movimento humano. Isso porque, dentre os resultados obtidos no presente estudo, os índices de internação por DCNT musculoesqueléticas e do sistema nervoso no ano de 2014 diminuíram de forma significativa entre os indivíduos de 50 a 59 anos e de 60 a 69 anos, quando comparados aos idosos de 80 anos ou mais.

Miranda et al. ${ }^{13}$, em um estudo, realizaram a caracterização de um grupo de promoção da saúde enfatizando o perfil epidemiológico das DCNT e a satisfação com o programa. A média de idade desses indivíduos estava entre 51 e 60 anos, sendo que as principais DCNT apresentadas eram hipertensão arterial (41\%) e artrose (35,89\%). Isso implica numa hipótese de transição do tipo de DCNT mais prevalente de acordo com a idade entre os achados do nosso estudo no que se refere as DCNT musculoesqueléticas, já que na faixa etária de 50 a 59 anos, os índices de internação foram mais altos quando comparados às faixas etárias de 40 a 49 anos e 70 a 79 anos no ano de 2015. Nesse estudo, realizado por Miranda et al. ${ }^{13}$, foi possível apontar, ainda, os benefícios que um programa de promoção da saúde pode proporcionar, tanto fisiológica quanto cognitivamente, e potencializar a qualidade de vida dos idosos. Além disso, ele salienta a importância da implementação da atividade física como um recurso eficiente no controle e avanço das DCNT no decorrer do envelhecimento.

Essa compreensão indica que é possível preservar a saúde e potencializar o envelhecimento saudável a partir de programas de promoção de atividade física, a fim de evitar o sedentarismo e prevenir o desenvolvimento de 
doenças crônicas ${ }^{14}$. Vale ressaltar que o desenvolvimento das DCNT pode ser classificado como riscos não modificáveis e modificáveis. Os fatores não modificáveis incluem a idade, havendo relação entre o envelhecimento e o risco de desenvolver DCNT, a hereditariedade, o sexo e a raça. Em relação aos fatores modificáveis, encontram-se a hipertensão arterial, a ingestão excessiva de álcool, o diabetes mellitus, obesidade, estresse, colesterol elevado e, também, salientase o sedentarismo ${ }^{15}$. Levando em consideração os fatores de risco modificáveis é possível perceber que as DCNT não são patologias inevitáveis ao envelhecimento. Isso quer dizer que elas podem ser prevenidas por meio do aprimoramento de ações de promoção e prevenção para esse perfil de população, como também a investigação do curso das patologias e seus fatores de riscos associados ao longo dos anos de tal forma que fomente o fortalecimento dessas ações ${ }^{3}$.

Com base no modelo do sistema do movimento humano apresentado por Sahrmann ${ }^{6}$ e nos resultados encontrados neste estudo, pode-se inferir que o desenvolvimento das DCNT no envelhecimento apresenta um curso de evolução e agravamento das incapacidades nos idosos. Com o embasamento teórico da autora Sahrmann ${ }^{6}$, os sistemas efetores do movimento, no caso, musculoesquelético e nervoso, são os que produzem o movimento visível de forma intencional pelo indivíduo. Já os sistemas de apoio do movimento, no caso, cardiovascular, respiratório e endócrino, são os que darão sustentação e manutenção do movimento, ou seja, é um suporte aos efetores de uma forma involuntária. Essa análise do movimento humano pode ser uma das explicações cabíveis para o curso das DCNT no passar da idade. Ao passo que a idade vai aumentando (70 a 79 anos e 80 anos ou mais), as doenças musculoesqueléticas e do sistema nervoso diminuem nos índices de internação, resultado esse apresentado no presente estudo. Da mesma forma, estudos como o de Alves et al. ${ }^{7}$, Pereira, Nogueira e da Silva ${ }^{12}$ e Miranda et al. ${ }^{13}$ mostram que as DCNT em indivíduos com mais de 70 anos são, principalmente, as cardiovasculares. Doenças essas que acometem um dos sistemas de apoio do movimento humano de Sahrmann ${ }^{6}$. Sendo assim, mais estudos envolvendo os índices de internação das DCNT que comprometem o sistema de apoio do movimento humano em idosos devem ser realizados com o intuito de verificar se o embasamento teórico da autora pode fomentar o estudo do desenvolvimento das DCNT.

Para buscar um envelhecimento saudável, a conscientização da população por meio de programas de prevenção de DCNT se faz indispensável. Deve-se investir no planejamento de políticas públicas que fomentem a elaboração de planos de ação capazes de minimizar os fatores de riscos modificáveis como o tabagismo, alcoolismo e sedentarismo ${ }^{16}$. Dessa forma, a caracterização da vigilância em saúde em relação às DCNT também implica no aprimoramento e implementação de políticas públicas voltadas para a promoção da saúde, especialmente norteando a assistência à saúde da população em processo de envelhecimento, a fim de operacionalizar o planejamento de assistência. Com isso, o delineamento epidemiológico da distribuição, magnitude e exposição da população aos fatores de risco, além da identificação dos condicionantes sociais, econômicos e ambientais, são essenciais para o planejamento e execuções dessas ações ${ }^{15}$. Consequentemente leva à redução dos gastos públicos com essas patologias, que podem ser evitadas ${ }^{14}$. 
Ademais, a partir do presente estudo pode-se inferir que o desenvolvimento das DCNT no processo de envelhecimento acarreta em sérios problemas de saúde no que tange à qualidade de vida do indivíduo e à economia do país. Corroborando com o estudo de Miranda et al. ${ }^{13}$, o acometimento por essas patologias influencia diretamente na independência dos idosos e, por isso, mais estudos que visem à elaboração de programas de promoção da saúde e prevenção de DCNT devem ser realizados com o intuito de diminuir os riscos modificáveis e, assim, maximizar o controle dessas patologias.

Vale salientar que, por ser uma amostra de uma base de dados, acreditamos que haja subnotificação desses dados, vista pela ausência de diferença estatística nos anos de 2011, 2012 e 2013. Entretanto, apesar da limitação descrita, é importante ressaltar que essas fontes têm um grande potencial para a condução de estudo exploratórios, assim como a possibilidade de comparação ao longo do tempo17.

Com isso, é visto que, a partir da ferramenta DATASUS, foi possível analisar os dados de internação hospitalar (HUSM) por DCNT que acometem o sistema musculoesquelético e sistema nervoso nos anos de 2008 a 2015. Tendo como conclusão o padrão de maior número dessas internações nas faixas etárias mais jovens em comparação com as faixas etárias mais velhas, o que implica na veracidade de que o modelo de sistema do movimento humano apresentado por Sahrmann6 pode ser um dos caminhos para mais estudos sobre a prevalência de DCNT no envelhecimento.

\section{REFERÊNCIAS}

1. Kernkamp CL, Costa CKF, Massuda EM, Silva ES, Yamaguchi UM, Bernuci, MP. Perfil de morbidade e gastos hospitalares com idosos no Paraná, Brasil, entre 2008 e 2012. Cad. Saúde Pública, 2016, 32(7): e00044115.

2. Pedrazzi EC, Rodrigues RAP, Schiaveto FV. Morbidade Referida e Capacidade Funcional De Idosos. Cienc Cuid Saude, 2007, 6(4): 407-413.

3. Silva JVF, Rodrigues APRA, Miyazawa AP. A relação entre o envelhecimento populacional e as doenças crônicas não transmissíveis: sério desafio de saúde pública. Ciên. Biológicas e da Saúde, 2015, 2(3): 91-100.

4. Duncan BB, Chor D, Aquino EML, Bensenor IM, Mill JG, Schmidt MI, Lotufo PA, Vigo A, Barreto SM. Doenças crônicas não transmissíveis no Brasil: prioridade para enfrentamento e investigação. Rev. Saúde Pública. 2012, 46(1): 126-134. 
5. APTA, American Physical Therapy Association. Physical Therapist Practice and The Human Movement System - White Paper. August, 2015.

6. Sahrmann SA. The Human Movement System: Our Professional Identity. Phys. Ther. 2014, 94:1034-1042.

7. Alves LC, Leimann BCQ, Vasconcelos MEL, Carvalho MS, Vasconcelos AGG, Da Fonseca TCO, Lebrão MC, Laurenti R. A influência das doenças crônicas na capacidade funcional dos idosos do Município de São Paulo, Brasil. Cad. Saúde Pública, 2007, 23(8):1924-1930.

8. Andrade LT, Chianca TCM. Validação de intervenções de enfermagem para pacientes com lesão medular e mobilidade física prejudicada. Rev. bras. enferm. 2013, 66(5): 688-693.

9. Costa AGS, Oliveira ARS, Alves FEC, Chaves DBR, Moreira RP, Araujo TL. Diagnóstico de enfermagem: mobilidade física prejudicada em pacientes acometidos por acidente vascular encefálico. Rev Esc Enferm. 2010, 44(3): 753-8.

10. Pereira AGS, Santos CT, Menegon DB, Mello B, Azambuja F, Lucena AF. Mapeamento de cuidados de enfermagem com a NIC para pacientes em risco de úlcera por pressão. Rev Esc Enferm. 2014, 48(3): 454-61.

11. Chibante CLP, Santo FHE, Santos TD, Pestana LC, Santos ACS, Pinheiro FM. Fatores associados à internação hospitalar em clientes com doenças crônicas. Cienc Cuid Saude. 2015, 14(4):1491-1497.

12. Pereira DS, Nogueira JAD, Silva CAB. Qualidade de vida e situação de saúde de idosos: um estudo de base populacional no Sertão Central do Ceará. Rev. Bras. Geriatr. Gerontol. 2015, 18(4):893-908.

13. Miranda AKP, Junior JÁ, Carvalho ALA, Rodrigues ZMR. Doenças crônicas não transmissíveis em usuários de um programa de atividade física de São Luís - MA. Hygeia. 2016, 12(23): 100 - 110.

14. Bielemann RM, Knuth AG, Hallal PC. Atividade física e redução de custos por doenças crônicas ao Sistema Único de Saúde. Rev. Bras. de Atividade Física \& Saúde. 2010, 15(10): 9-14. 
15. Casado L, Vianna LM, Thuler LCS. Fatores de risco para doenças crônicas não transmissíveis no Brasil: Uma revisão sistemática. ver. Bras. de Cancerologia. 2009, 55(4): 379-388.

16. Virtuoso JF, Balbé GP, Mazo GZ, Pereira MGS, Santos FS. Morbidade e mortalidade da população idosa de Florianópolis: um estudo comparativo entre homens e mulheres. Rev. Bras. Geriatr. Gerontol. 2010, 13(2): 215-223.

17. Costa MFFL, Guerra HL, Barreto SM, Guimarães RM. Diagnóstico da situação da população idosa brasileira: um estudo da mortalidade e das internações hospitalares públicas. Inf Epidemiol SUS. 2000, 9: $23-41$. 